\title{
A Vibration-Based PMN-PT Energy Harvester
}

\author{
Alex Mathers, Kee S. Moon, and Jingang Yi, Senior Member, IEEE
}

\begin{abstract}
We report design, modeling, analysis, and experimental study of a vibration-based piezoelectric energy harvester. The energy harvester is made of a composite cantilever of a single crystal relaxor ferroelectric material, $(1-x) \mathrm{Pb}\left(\mathrm{Mg}_{1 / 3} \mathrm{Nb}_{2 / 3}\right) \mathrm{O}_{3-x} \mathrm{PbTiO}_{3}$ (PMN-PT), and a polydimethylsiloxane (PDMS) base layer. A PDMS proof mass is constructed at the tip of the composite cantilever beam and is used as a means to tune the system natural frequency. The use of the PMN-PT piezoelectric material and an interdigited electrodes (IDEs) design improves the energy conversion efficiency. A dynamic systems modeling approach is employed to analyze the responses and the performance of the harvester design. We have demonstrated that a prototype of the harvester with a size of $7.4 \mathrm{~mm} \times 2 \mathrm{~mm} \times 110 \mu \mathrm{m}$ outputs a voltage of $10 \mathrm{~V}$ $(0.3 \mathrm{~mW}$ power) under a vibration excitation with a peak-to-peak amplitude of $1 \mathrm{~mm}$ at a frequency around $1.3 \mathrm{kHz}$. Based on the experimental results, the power density prediction of the proposed harvester design shows a superior performance than that of the other reported piezoelectric harvesters.
\end{abstract}

Index Terms-Composite cantilever beam, energy harvester, interdigited electrodes (IDEs), piezoelectric harvester, PMN-PT.

\section{INTRODUCTION}

$\mathbf{O}$ VER the past decade, there has been a significant growth in the applications of wireless networked sensors and smart devices, such as embedded sensors in buildings, medical implant devices, and wireless automotive tire sensors, etc. Long-term supply of power to these smart devices is challenging due to their being embedded in the host structure and the limited lifetime of conventional electrochemical batteries. Energy harvesting from ambient environments provides a perpetual power solution for these devices [1].

Converting environmental vibration into electric energy has been actively explored with various piezoelectric materials and designs [2]-[12]. Most of the vibration energy converters are based on a design in which the natural frequency of the vibration system is close to the frequency of the vibrational excitation source. One of the challenges of the resonant-based energy converter is the energy harvesting efficiency. In [7], the effective-

Manuscript received October 15, 2008; accepted December 23, 2008. Current version published May 20, 2009. This paper was presented in part at the 2008 ASME Dynamic Systems and Control Conference, Ann Arbor, MI, USA, October 19-22, 2008. The associate editor coordinating the review of this paper and approving it for publication was Prof. Gianluca Lazzi.

A. Mathers was with the Department of Mechanical Engineering, San Diego State University, San Diego, CA 92182 USA. He is now with Qualcomm Inc., San Diego, CA 92121 USA.

K. S. Moon is with the Department of Mechanical Engineering, San Diego State University, San Diego, CA 92182-1323 USA (e-mail: kmoon@mail.sdsu. edu).

J. Yi is with the Department of Mechanical and Aerospace Engineering, Rutgers University, Piscataway, NJ 08854-8058 USA (e-mail: jgyi@rutgers.edu). Address all correspondence to J. Yi.

Color versions of one or more of the figures in this paper are available online at http://ieeexplore.ieee.org.

Digital Object Identifier 10.1109/JSEN.2009.2021192 ness of vibration-based energy harvesting is discussed for various energy conversion technologies, such as electromagnetic, piezoelectric, and electrostatic methods. It has been shown that coupling coefficient of the piezoelectric material has a significant impact on the effectiveness of energy harvesting. Recently, numerous attempts have been made to increase the efficiency of piezoelectric energy harvesting devices by using new piezoelectric materials and innovative electrode designs [6], [7]. For example, in [6], the $d_{33}$ mode of the piezoelectric transducer is used to generate about 20 times higher voltage than that of the $d_{31}$ mode of the same harvester geometry.

In this paper, we present design, modeling, analysis, and experimental study of a vibration-based energy harvester that is made of single crystal relaxor ferroelectric material. The main contributions of this study are twofolds. First, we take several novel approaches to improve the energy conversion efficiency. We use a piezoelectric material, $(1-x) \mathrm{Pb}\left(\mathrm{Mg}_{1 / 3} \mathrm{Nb}_{2 / 3}\right) \mathrm{O}_{3}-x \mathrm{PbTiO}_{3}$ (PMN-PT), as the energy conversion component. PMN-PT has much higher coupling coefficient and electromechanical coefficient, which improve the efficiency of the energy conversion [13]-[15]. We design and fabricate interdigited electrodes (IDEs) on the PMN-PT layer. The use of IDEs improves the output voltage significantly. Furthermore, the construction of the proof mass at the cantilever tip is used as a means to tune the natural frequency of the harvester design. Second, we take a dynamic system modeling approach to analyze the harvester design and performance. The analytical formulation provides guidances for the beam/mass harvester design. We also quantitatively investigate and compare the harvester power density performance with the other harvester designs using the dynamic system modeling scheme.

This paper is organized as follows. The energy harvester design and prototype is presented in Section II. We present a dynamic system model for the vibration-based cantilever harvester in Section III. Model validation and harvester performance are presented in Section IV. We compare the harvester performance with an MEMS-based PZT harvester design in Section V before concluding this paper in Section VI.

\section{PMN-PT/PDMS-BASED ENERGy HARVESTER}

We use a single crystal relaxor ferroelectric material, PMN-PT, as the energy harvester material. For PMN-PT, the piezoelectric coefficient $\left(d_{33}>1500 \mathrm{pC} / \mathrm{N}\right)$, dielectric constant $\left(k_{R T} \approx 3000\right)$, and electromechanical coupling factor $\left(k_{33} \approx 92 \%\right)$ are significantly higher than those of the lead zirconate titanate $\mathrm{Pb}\left(\mathrm{Zr}_{1-x} \mathrm{Ti}_{x}\right) \mathrm{O}_{3}(\mathrm{PZT})\left(d_{33} \approx 400 \mathrm{pC} / \mathrm{N}\right.$, $k_{33} \approx 70 \%$ ) [16], [17]. The IDE design takes advantages of transverse mode (e.g., $d_{33}$ ) to increase the efficiency of energy harvesting.

We use polydimethylsiloxane (PDMS) (Dow Corning Slygaard 184) to form a coating layer on the PMN-PT cantilever and to fabricate a proof mass at the cantilever tip. PDMS is an 


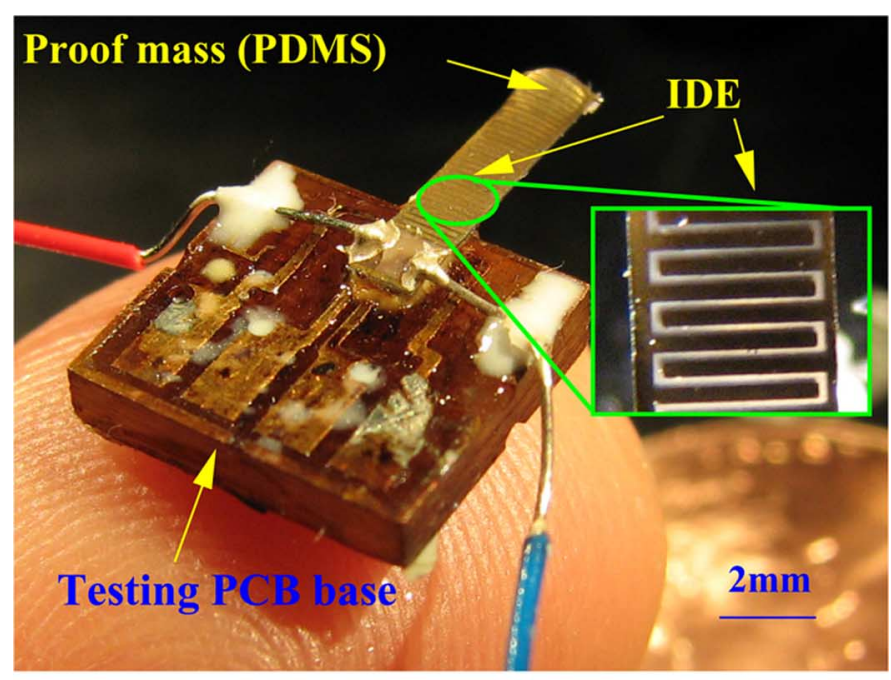

(a)

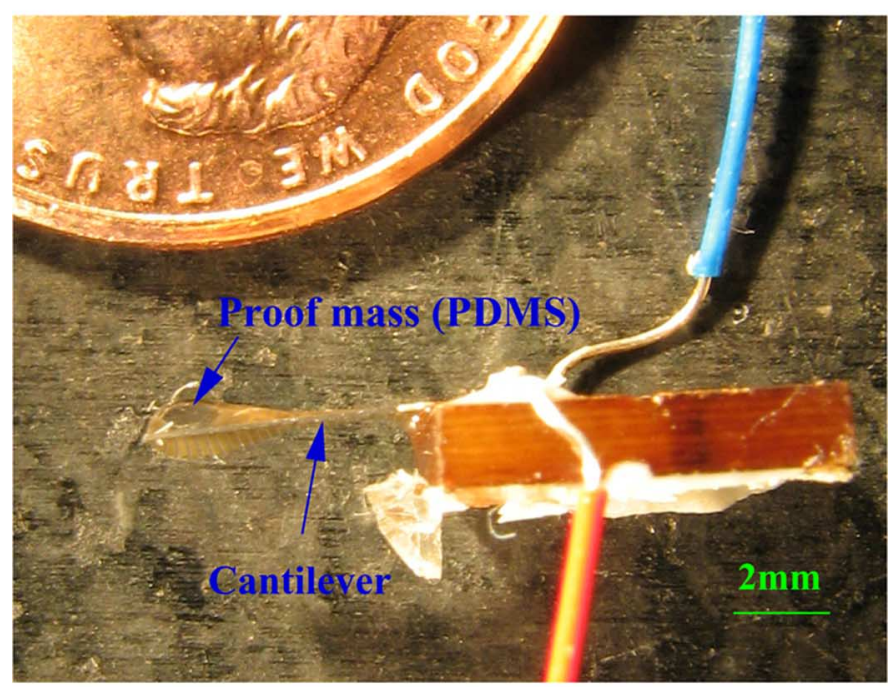

(b)

Fig. 1. A PMN-PT/PDMS energy harvester. (a) Top view. (b) Side view.

inert, clear organic polymer. What makes PDMS attractive for the harvester prototype is its ability to make conformal contact with surface along with its chemical and thermal stability. The main purpose of fabricating the PDMS coating layer is to reduce the stress concentration at the support and therefore to increase the reliability of the harvester. Although we have not quantitatively studied the long-term PMN-PT/PDMS adhesion reliability, we have however observed that the use of the PDMS layer significantly improves lifetime of the PMN-PT harvester.

To fabricate the harvester prototype, we use $\langle 001\rangle$-factory-oriented PMN-PT single crystal plates with a thickness of $110 \mu \mathrm{m}$. The PMN-PT thin plates are undergone a mechanical polishing and thin layers $(50 \mathrm{~nm})$ of Gold are deposited on the top and the bottom sides to form fine primary electrode layers. The interdigitated electrode patterns are custom micromachined by photolithography processes. A pair of $50 \mu \mathrm{m}$-gap periodic electrodes (100 $\mu \mathrm{m}$ wide) are fabricated on the bottom and the top of the PMN-PT layer [Fig. 1(a)]. A PMN-PT harvester prototype is fabricated with a dimension of $7.4 \mathrm{~mm} \times 2 \mathrm{~mm} \times 110 \mu \mathrm{m}$. The PMN-PT cantilever is then attached to a PCB board $(8 \mathrm{~mm} \times 8 \mathrm{~mm} \times 1 \mathrm{~mm})$, as shown in Fig. 1.

To coat a PDMS layer, we dip the PMN-PT cantilever into PDMS. Any air cavities that may be trapped are carefully removed. The PMN-PT/PDMS cantilever is then heated to a temperature around $100{ }^{\circ} \mathrm{C}$ for about $15 \mathrm{~min}$. Once the PDMS has cured and hardened, it is cooled down to room temperature. The next step is to add the proof mass. We only dip the cantilever's tip into PDMS. The amount of PDMS applied can be controlled by multiple quick dippings. After a satisfactory amount of PDMS is applied to the cantilever's tip, the cantilever is heated again to a temperature around $100^{\circ}$ and cooled down as before. The PMN-PT/PDMS cantilever is under poling for about one hour in a $100 \mathrm{~V}$ dc electric field.

In this study, silicone-based PDMS is used as proof mass because of its ease fabrication in room temperature, easily bonding to itself to the PMN-PT layer, and thus convenient control of its weight. We have first attempted to attach heavier metal pieces at the cantilever tip and it failed because of the fragility of the PMN-PT cantilever beam. Since the focus of this paper is on the conceptual design, development, and analysis of the PMN-PT energy harvester, we do not consider the possible long-term adhesion issue between the PMN-PT and PDMS layers and the temperature effect on the harvester performance. We will quantitatively investigate these subjects in the future work.

\section{HARVESTER MODELING}

In this section, we develop a dynamic system modeling approach to analytically capture the harvester's responses and performance under excitations. Comparing with other modeling approaches, such as finite-element analysis, the dynamic system modeling can provide a closed-form solution that can be used directly to guide the harvester design and performance analysis.

\section{A. Dynamic Models of the Cantilever Harvester}

The harvester prototype is considered as a composite cantilever with a point mass on its tip; see Fig. 2. The following assumptions are made for modeling the composite cantilever structure: (1) The proof mass and the beam support are rigid; (2) The strain across the bonding of the PMN-PT/PDMS layers is continuous; (3) Both the PMN-PT and PDMS beams are elastic and obey Hooke's law; (4) The material of PMN-PT layer is orthotropic; and (5) With the support displacement $u(t)$, the beam is under pure bending and the stress in the $z$ and $y$ directions are negligible.

The length and width of the PMN-PT/PDMS composite cantilever are denoted by $l$ and $b$, respectively. The mass of the proof mass is denoted by $M_{p}$. The heights of the PMN-PT and PDMS layers are denoted by $h_{p}$ and $h_{b}$, respectively. The mass density per unit length for the PMN-PT and the base beams are denoted by $\rho_{p}$ and $\rho_{b}$, respectively.

We consider the composite layer of the PMN-PT and PDMS as one beam with flexural rigidity $E I_{\text {eq }}$ (we will calculate $E I_{\text {eq }}$ later in this section). The mass density per unit length for the composite beam is $m:=\left(\rho_{p} h_{p}+\rho_{b} h_{b}\right) /\left(h_{p}+h_{b}\right)$. We consider the dynamics of the beam-mass system under an excitation $u(t)$. The vertical deflection of the beam is denoted by $w(x, t)$ at location $x$ and time $t$ in the coordinate system $x o z$ located on 


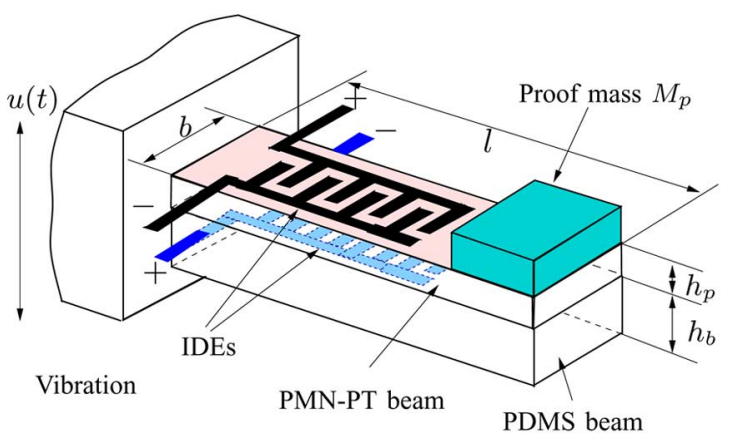

(a)


(b)

Fig. 2. (a) Operating diagram of the PMN-PT/PDMS harvester with IDEs. (b) A dimensional schematic of the composite cantilever.

the base (Fig. 2). The kinetic and potential energy of the system are, respectively, as

$$
\begin{aligned}
T= & \frac{1}{2} \int_{0}^{l} m(\dot{w}(x, t)+\dot{u}(t))^{2} d x \\
& +\frac{1}{2} M_{p}(\dot{w}(l, t)+\dot{u}(t))^{2} \\
V= & \frac{1}{2} \int_{0}^{l} E I_{\mathrm{eq}}\left(w^{\prime \prime}\right)^{2} d x
\end{aligned}
$$

where $\dot{w}(x, t):=\partial w(x, t) / \partial t$ and $w^{\prime \prime}(x, t):=\partial^{2} w(x, t) / \partial x^{2}$. If we denote the viscous damping coefficient for the composite beam is $c$, then the virtual work done by the damping force is

$$
\delta W_{c}=-\int_{0}^{l} c \dot{w}(x, t) \delta w d x
$$

where $\delta w$ is the virtual displacement. Using the extended Hamilton's principles

$$
\int_{t_{1}}^{t_{2}}\left(\delta T-\delta V+\delta W_{c}\right) d t=0
$$

we obtain the equations of motion and boundary conditions

$$
\begin{aligned}
& m \frac{\partial^{2} w}{\partial t^{2}}+c \frac{\partial w}{\partial t}+E I \frac{\partial^{4} w}{\partial x^{4}}=-m \ddot{u} \\
& M_{p} \frac{\partial^{2} w(l, t)}{\partial t^{2}}-E I \frac{\partial^{3} w(l, t)}{\partial x^{3}}=-M_{p} \ddot{u} \\
& w(0, t)=w^{\prime}(0, t)=w^{\prime \prime}(l, t)=0 .
\end{aligned}
$$

We define

$$
\xi:=\frac{x}{l}, \quad \alpha:=\frac{M_{p}}{m l}, \quad \lambda^{4}:=\frac{m l^{4} \omega^{2}}{E I_{\mathrm{eq}}}
$$

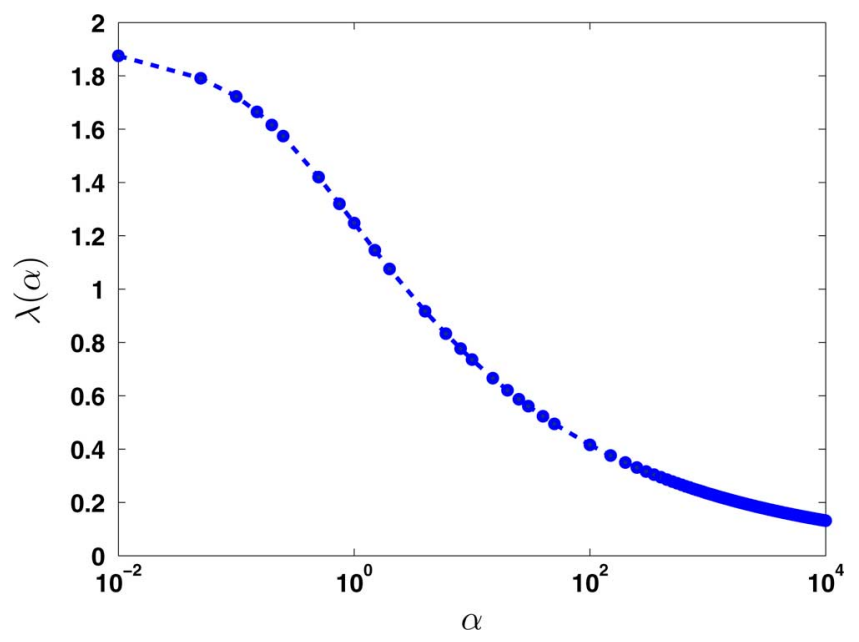

Fig. 3. A relationship between the natural frequency parameter $\lambda(\alpha)$ and the mass ratio $\alpha$.

as the dimensionless length of the beam, mass ratio of the proof mass and the composite beam, and the natural frequency parameters of the beam-mass system, respectively. The characteristic equation of the natural frequency of the dynamic systems given by (2) is then obtained with $c=0$ as follows:

$$
1+\cos \lambda \cosh \lambda+\lambda \alpha(\cos \lambda \sinh \lambda-\sin \lambda \cosh \lambda)=0
$$

For various $\alpha$, we solve the above equation numerically. Fig. 3 shows the values of $\lambda$ as a function of $\alpha$, namely, $\lambda:=\lambda(\alpha)$.

\section{B. Harvester Output Models}

The cross section of the composite beam is shown in Fig. 2(b). The coordinate system xoz in Fig. 2(b) is setup with the origin at the neural axis $N N^{\prime}$. Assuming the distance between the bonding layer and the neutral axis is $a$, we calculate the value of $a$ for the composite beam under a bending load. For the PMN-PT layer, the strain $\sigma_{1 p}$ and stress $\epsilon_{1 p}$ along the $x$ axis direction can be written as $\sigma_{1 p}=E_{p} \epsilon_{1 p}$, where $E_{p}$ is the elastic modulus of the PMN-PT materials. Similarly, for the PDMS layer, the stress $\sigma_{1 b}$ and strain $\epsilon_{i b}$ are related to each other as $\sigma_{1 b}=E_{b} \epsilon_{1 b}$, where $E_{b}$ is the elastic modulus of the PDMS material. The strain along the $x$ axis direction at location $x$ can be obtained as $\epsilon_{1}=z / R(x)$, where $z$ is the vertical coordinate of the cross section and $R(x)$ is the deflection radius of the beam at location $x$. The force balances at the cross section of the beam at location $x$ are then written as

$$
\begin{aligned}
& \int_{-\left(h_{b}-a\right)}^{a} E_{b} b \frac{z}{R} d z+\int_{a}^{h_{p}+a} E_{p} b \frac{z}{R} d z=0 \\
& \int_{-\left(h_{b}-a\right)}^{a} E_{b} b \frac{z}{R} z d z+\int_{a}^{h_{p}+a} E_{p} b \frac{z}{R} z d z=M(x)
\end{aligned}
$$

where $M(x)$ is the bending moment at location $x$. Solving the above equation, we obtain

$$
a=\frac{1}{2} \frac{E_{b} h_{b}^{2}-E_{p} h_{p}^{2}}{E_{b} h_{b}+E_{p} h_{p}}, \quad \frac{1}{R(x)}=\frac{M(x)}{E I_{\mathrm{eq}}}=\frac{\partial^{2} w}{\partial x^{2}}
$$


where

$$
\begin{aligned}
E I_{\mathrm{eq}}=\frac{1}{3} b E_{b}\left(h_{b}^{3}-3 a h_{b}^{2}+3 a^{2} h_{b}\right) & \\
& +\frac{1}{3} b E_{p}\left(h_{p}^{3}+3 a h_{p}^{2}+3 a^{2} h_{p}\right) .
\end{aligned}
$$

The IDEs are located on the top and bottom surfaces of the PMN-PT layer. Therefore, the average stress in the PMN-PT layer is

$$
\bar{\sigma}_{1 p}(x)=E_{p} \frac{1}{R(x)}\left(\frac{h_{p}}{2}+a\right)=E_{p}\left(\frac{h_{p}}{2}+a\right) \frac{\partial^{2} w}{\partial x^{2}} .
$$

We use the generalized single-degree-of-freedom (SDOF) approximation [11], [18] for the solution of dynamic equations given by (2). By variable separation, we write $w(x, t)=\phi(x) q(t)$, where $\phi(x)$ is the modal shape and $q(t)$ is the generalized coordinate for the SDOF system. We consider the following mode shape $\phi(x)$ for the beam-mass systems

$$
\phi(x)=\sin (\lambda \xi)-\sinh (\lambda \xi)-B(\xi)[\cos (\lambda \xi)-\cosh (\lambda \xi)]
$$

where $\xi$ is defined in (3) and

$$
B(\xi)=\frac{\sin (\lambda \xi)+\sinh (\lambda \xi)}{\cos (\lambda \xi)+\cosh (\lambda \xi)}
$$

Furthermore, using expansions for hyperbolic functions, $\phi(x)$ can be approximated as

$$
\phi(x)=A \xi^{2}-C \xi^{3}
$$

where

$$
A:=A(\alpha)=\frac{\sin \lambda+\sinh \lambda}{\cos \lambda+\cosh \lambda} \lambda^{2}, C:=C(\alpha)=\frac{1}{3} \lambda^{3} .
$$

Using the generalized SDOF system and extended Hamilton's principles (1), the equation of motion for the beam-mass systems in terms of $q(t)$ is obtained as

$$
m_{e} \ddot{q}(t)+c_{e} \dot{q}(t)+k_{e} q(t)=-f_{e} \ddot{u}(t)
$$

where generalized mass $m_{e}$, generalized damping coefficient $c_{e}$, generalized stiffness coefficient $k_{e}$, and generalized force coefficient $f_{e}$ are, respectively, as

$$
\begin{aligned}
m_{e} & =\int_{0}^{l} m \phi^{2}(x) d x+M_{p} \phi^{2}(l)=a_{1} M_{p}+a_{2} m l \\
c_{e} & =\int_{0}^{l} c \phi^{2}(x) d x=a_{2} c l \\
k_{e} & =\int_{0}^{l} E I_{\mathrm{eq}}\left(\phi^{\prime \prime}(x)\right)^{2} d x=\frac{4 E I_{\mathrm{eq}}}{l^{3}} a_{3} \\
f_{e} & =\int_{0}^{l} m \phi(x) d x+M_{p} \phi(l)=a_{4} M_{p}+a_{5} m l
\end{aligned}
$$

and

$$
\begin{aligned}
& a_{1}=(A-C)^{2}, a_{2}=\frac{A^{2}}{5}-\frac{A C}{3}+\frac{C^{2}}{7} \\
& a_{3}=A^{2}-3 A C+3 C^{2}, a_{4}=A-C, a_{5}=\frac{A}{3}-\frac{C}{4} .
\end{aligned}
$$

Note that the values of the above $a_{i}$ 's depend on the variable $\alpha$ due to the relationships (9).

The charge generated by the PMN-PT/PDMS cantilever under the excitation $u(t)$ is obtained as

$$
\begin{aligned}
Q(t) & =\int_{0}^{l} d_{33} \bar{\sigma}_{1 p}(x) b d x \times \int_{0}^{l} d_{33} b E_{p}\left(\frac{h_{p}}{2}+a\right) \frac{\partial^{2} w}{\partial x^{2}} d x \\
& =d_{33} b E_{p}\left(\frac{h_{p}}{2}+a\right) q(t) \int_{0}^{l} \phi^{\prime \prime}(x) d x \\
& =\frac{d_{33} E_{p} b a_{6}}{2 a_{4} l}\left(h_{p}+2 a\right) w_{p}(t)
\end{aligned}
$$

where $w_{p}(t):=w(l, t)=\phi(l) q(t)$ is the cantilever tip (e.g., proof mass) displacement and $a_{6}=2 A-3 C$. If we consider an open circuit for the PMN-PT/PDMS harvester, then the output voltage $V_{o}$ can be calculated as

$$
V_{o}(t)=\frac{Q(t)}{C_{p}}=K w_{p}(t)
$$

where $C_{p}$ is the capacitance of the PMN-PT piezoelectric harvester and constant

$$
K:=\frac{d_{33} E_{p} b a_{6}}{2 a_{4} C_{p} l}\left(h_{p}+2 a\right) .
$$

From (10), (14), and relationship $w_{p}(t)=a_{4} q(t)$, we find the transfer function between the excitation displacement $u(t)$ and the open-circuit voltage $V_{o}(t)$ as

$$
G_{v}(s):=\frac{V_{o}(s)}{U(s)}=-\frac{A_{v} \omega_{n}^{2} s^{2}}{s^{2}+2 \zeta \omega_{n} s+\omega_{n}^{2}}
$$

where

$$
\omega_{n}^{2}=\frac{k_{e}}{m_{e}}, 2 \zeta \omega_{n}=\frac{c_{e}}{m_{e}}, A_{v}=\frac{K a_{4} f_{e}}{k_{e}} .
$$

A similar transfer function $G_{p}(s)$ between the base displacement $u(t)$ and the tip displacement $w_{p}(t)$ is obtained as

$$
G_{p}(s):=\frac{W_{p}(s)}{U(s)}=-\frac{A_{p} \omega_{n}^{2} s^{2}}{s^{2}+2 \zeta \omega_{n} s+\omega_{n}^{2}}
$$

where $A_{p}:=A_{v} / K=a_{4} f_{e} / k_{e}$.

Under a harmonic excitation, both $G_{p}(s)$ and $G_{v}(s)$ achieve their maximum values of magnitudes at frequency $\omega=\omega_{n} / \sqrt{1-2 \zeta^{2}}$ and the maximum values of output voltage and proof mass displacement are, respectively

$$
\begin{aligned}
\left|V_{0}\right|_{\max } & =\frac{A_{v} \omega_{n}^{2} u_{p}}{2 \zeta \sqrt{1-\zeta^{2}}} \\
\left|w_{p}\right|_{\max } & =\frac{A_{p} \omega_{n}^{2} u_{p}}{2 \zeta \sqrt{1-\zeta^{2}}}
\end{aligned}
$$

where $u_{p}$ is the peak amplitude of the harmonic excitation.

Remark 1: We do not consider the electromechanical coupling effects, such as cross-damping effects, in the above models. As we show in the next section, possibly due to the use of the PDMS layer, the estimated mechanical damping of the harvester prototype (with damping ratio $\zeta=0.123$ ) is fairly 


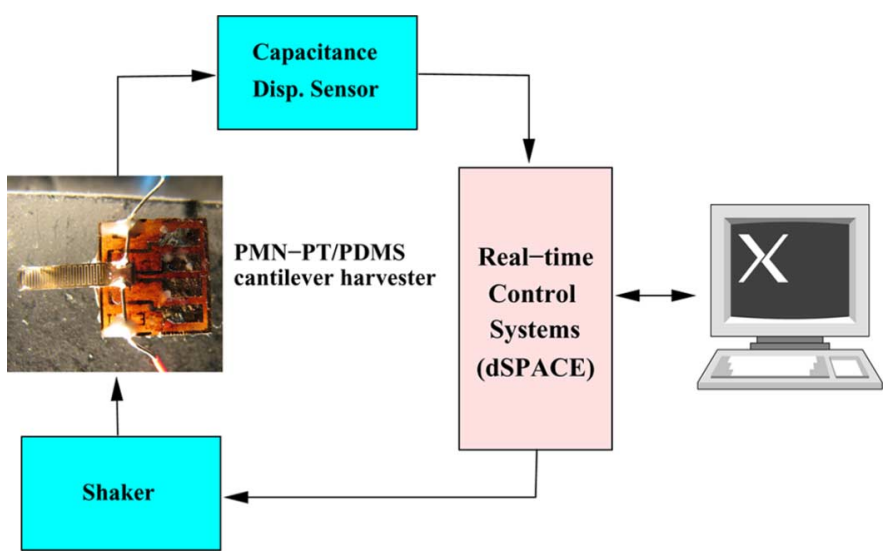

Fig. 4. A schematic of the harvester testing systems.

TABLE I

Geometry ANd Material Properties of CANTILEVER HaRvester Systems

\begin{tabular}{|c|c|c|c|c|c|}
\hline \hline & $l(\mathrm{~mm})$ & $b(\mathrm{~mm})$ & $h(\mu \mathrm{m})$ & $\rho\left(\mathrm{g} / \mathrm{cm}^{3}\right)$ & $E(\mathrm{GPa})$ \\
\hline PMN-PT & 7.4 & 2 & 120 & 7.7 & 105 \\
\hline PDMS & 7.4 & 2 & 102 & 1.06 & $3 \times 10^{-3}$ \\
\hline
\end{tabular}

large. Thus, we assume that the electromechanical coupling damping effect is negligible in the model.

\section{EXPERIMENTS}

\section{A. Experiment Setup}

To test the harvester system, we set up a testbed as shown in Fig. 4. The cantilever harvester is mounted on a vibration shaker. A high-precision capacitance sensor system (Lion Precision Inc.) is used to measure the tip displacement of the cantilever. A real-time control system (dSPACE ACE1104CLP) is used to control the motion of the shaker and also collect the displacement and output voltage measurement data from the harvester.

\section{B. Parameter Estimations}

The material geometric and property parameters are listed in Table I. Instead of directly measuring the thickness of the transparent PDMS layer, we estimate the thickness by using the weight difference before and after coating the PDMS layer. It has been found that the mass difference between and after coating is $1.6 \mathrm{mg}$. Using the mass density of the PDMS material, we calculate the thickness of the PDMS layer. By the same method, we estimate the mass of the PDMS proof mass is $1.3 \mathrm{mg}$. We then calculate the composite beam rigidity and mass density properties. The estimated parameters are listed in Table II.

With these estimated parameters, we solve (4) to obtain the natural frequencies of the PMN-PT/PDMS composite beammass systems. Since most excitations in environments are in a low-frequency range [8] and the largest amount of energy is around the first natural frequency $\omega_{1}$, we here only calculate the frequency responses around the first (fundamental) natural frequency. Table III shows the comparison of the analytical and
TABLE II

ESTIMATED Rigidity, MASS, AND FREQUENCY PARAMETERS OF THE CANTILEVER HARVESTER

\begin{tabular}{|c|c|c|c|c|}
\hline \hline$E I_{e q}\left(\mathrm{Nm}^{2}\right)$ & $m(\mathrm{~kg} / \mathrm{m})$ & $M_{p}(\mathrm{mg})$ & $\alpha$ & $\lambda(\alpha)$ \\
\hline $3.02 \times 10^{-5}$ & $1.1 \times 10^{-3}$ & 1.3 & 0.16 & 1.655 \\
\hline \hline
\end{tabular}

TABLE III

ESTIMATED AND MEASURED FundAMENTAL NATURAL FREQUENCY $\omega_{1}$ OF THE CANTILEVER HaRVESTER WITH AND Without the ProOf Mass

\begin{tabular}{|c|c|c|c|}
\hline \hline & Analytical Est. & Freq Scan & Step Resp. \\
\hline$\omega_{1}(\mathrm{~Hz})$ with $M_{p}$ & 1321 & 1340 & 1215 \\
\hline$\omega_{1}(\mathrm{~Hz})$ without $M_{p}$ & 1695 & 1760 & - \\
\hline \hline
\end{tabular}

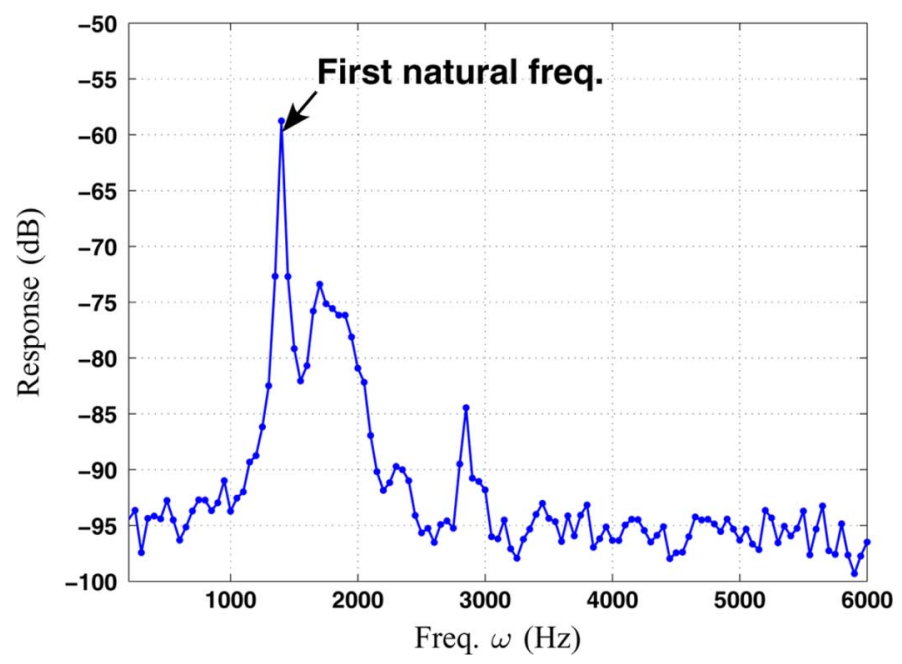

Fig. 5. A dynamic frequency response of the cantilever harvester.

experimental results of the estimates of $\omega_{1}$ for the composite cantilever with and without the proof mass. The analytical estimates of $\omega_{1}$ in both cases match the experimental results within a small error range.

The experimental dynamic frequency response of the cantilever harvester with the proof mass is shown in Figs. 5 and 7(a). It is clearly observed that the first natural frequency is around $1340 \mathrm{~Hz}$ [from Fig. 7(a)]. Note that the shifting resonant frequency as the increased input magnitude shown in Fig. 7(a) is due to the nonlinearity of the PMN-PT piezoelectric materials [19]. We do not consider such a nonlinearity in the model.

We use the dynamic system model to estimate the damping coefficient and the transfer function gains. Fig. 6 shows the step response of the displacement of the cantilever tip under a step excitation. The displacement measurements in Fig. 6 are relative to a fixed reference while the proof mass displacement $w_{p}(t)$ is calculated with respect to the moving base. Therefore, under the step excitation $u_{p}(t)=24 \mu \mathrm{m}, w_{p}(t)$ is expected to oscillate around the equilibrium at a distance of $24 \mu \mathrm{m}$ from its original location, as shown in Fig. 6. In Fig. 6, the input excitation is not a perfect step signal due to the imperfect performance of the vibration shaker under a step-shape input voltage. 


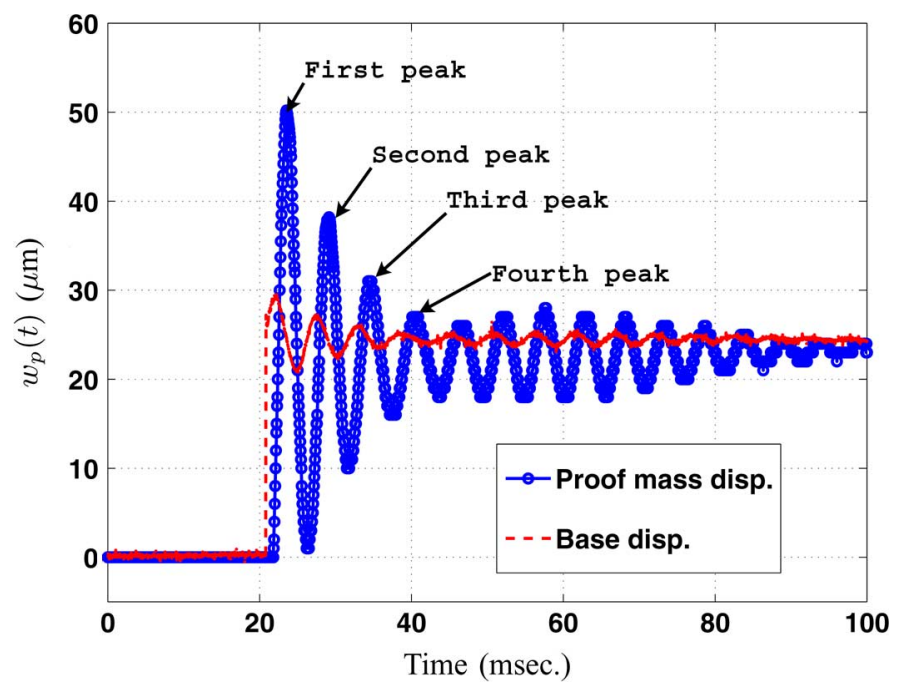

Fig. 6. A step response of the cantilever harvester.

From (18), under a step input $U(s)=u_{p} / s$, we have the displacement response of the cantilever tip

$$
w_{p}(t)=\frac{A_{p} \omega_{n}^{2} u_{p}}{\sqrt{1-\zeta^{2}}} e^{-\zeta \omega_{n} t} \cos \left(\omega_{d} t+\phi\right)
$$

where $\phi=\sin ^{-1} \zeta$. The $i$ th peak value of the step response $w_{p}(t)$ is denoted by $w_{p i}, i=1,2, \cdots$ Using $w_{p i}$, the damping ratio coefficient $\zeta$ and gain $A_{p}$ are estimated, respectively, as

$$
\begin{aligned}
\zeta_{i} & =\sqrt{1-\frac{1}{\left(\log R_{i} / 2 \pi\right)^{2}+1}} \\
A_{p i} & =\frac{w_{p i} \sqrt{1-\zeta^{2}}}{u_{p} \omega_{n}^{2} \cos \phi \exp \left(-\frac{\zeta(2 i \pi-2 \phi)}{\sqrt{1-\zeta^{2}}}\right)}
\end{aligned}
$$

where $R_{i}=w_{p i} / w_{p(i+1)}$ is the ratio of the adjacent peak values. The detailed derivation of the results in (20) is given in the Appendix.

We consider an average $\zeta=(1 / 3) \sum_{i=1}^{3} \zeta_{i}$ and an average $A_{p}=(1 / 3) \sum_{i=1}^{3} A_{p i} \mathrm{~m}$, and the estimated damping ratio coefficient $\zeta$ and gain $A_{p}$ are shown in Table IV. The estimated natural frequency (average through several oscillation cycles) using the step response is listed in Table III.

\section{Harvester Performance}

Fig. 7(a) shows the peak amplitude of the harvester output voltage (open circuit) versus the excitation frequency with three different peak amplitudes. Here, the input base vibration excitation is chosen to be sinusoidal. It is clearly observed an increase of the output voltage when the input excitation magnitude is increasing around $\omega_{1}=1340 \mathrm{~Hz}$. Fig. 7(b) shows the relationship between the peak amplitude $u_{p}$ and output voltage $V_{p}$, and a linear function is used to approximate the $V_{p}-u_{p}$ curve. Indeed, the analysis results in (19) show a linear relationship between $V_{p}$, namely, $\left|V_{0}\right|_{\max }$, and $u_{p}$.

The results in Fig. 7(b) show that the output voltage can reach $14 \mathrm{~V}$ (peak-to-peak) under the excitation with a peak-to-peak

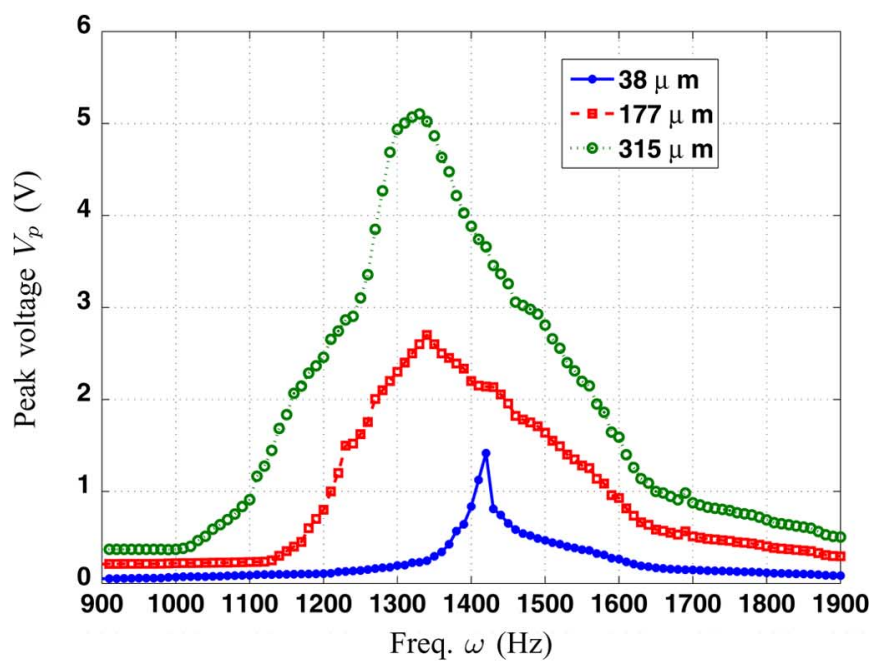

(a)

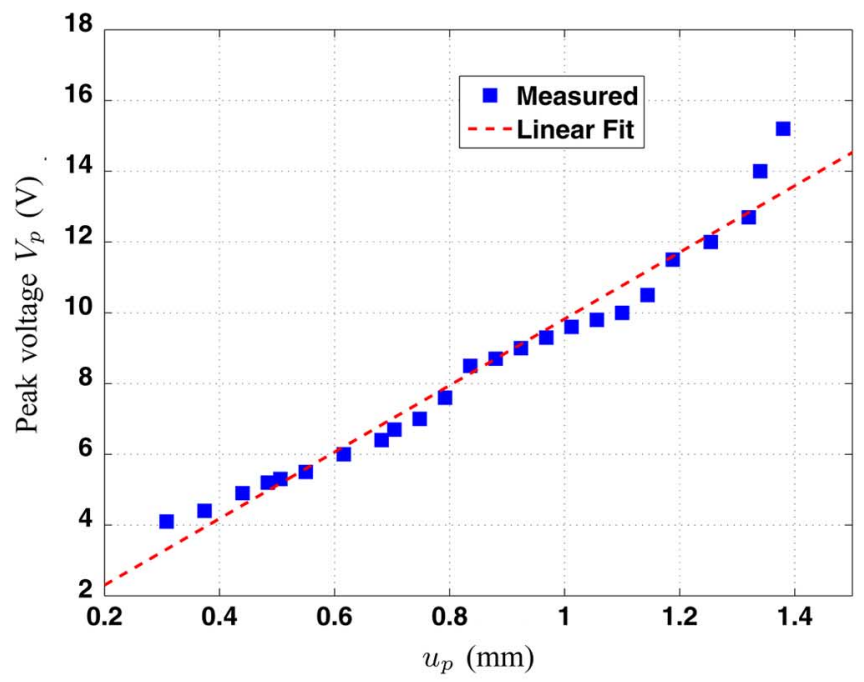

(b)

Fig. 7. (a) Harvest output voltage versus excitation frequency at three different base peak amplitudes. (b) Output voltage versus the base peak amplitudes around the first natural frequency.

TABLE IV

Estimated DyNAmic Model PARAMETERS FOR THE CANTILEVER HARVESTER

\begin{tabular}{|c|c|c|c|}
\hline \hline$\zeta$ & $A_{p}\left(\mathrm{~s}^{2} / \mathrm{rad}^{2}\right)$ & $A_{v}\left(\mathrm{Vs}^{2} / \mathrm{m} \cdot \mathrm{rad}^{2}\right)$ & $K(\mathrm{~V} / \mathrm{m})$ \\
\hline 0.123 & $1.937 \times 10^{-8}$ & $2.894 \times 10^{-5}$ & 1494 \\
\hline \hline
\end{tabular}

amplitude $1.4 \mathrm{~mm}$. With the data shown in Fig. 7(b) and relationship given in (19), we obtain the estimates of parameters $A_{v}$ and $K$ listed in Table IV. Therefore, we write the transfer function $G_{v}(s)$ as

$$
G_{v}(s)=-\frac{1876 s^{2}}{s^{2}+2041 s+6.89 \times 10^{7}}
$$

The harvester is then connected to an ac ampere meter to measure the short-circuit output current. Fig. 8(a) shows a relationship between peak amplitudes of the short-circuit current $I_{p}$ and $u_{p}$ around $\omega_{1}$. A clearly linear relationship between $I_{p}$ and $u_{p}$ is observed. Fig. 8(b) shows the calculated short-circuit output power (RMS) $P_{\text {rms }}$ versus the peak-amplitude $u_{p}$ around 


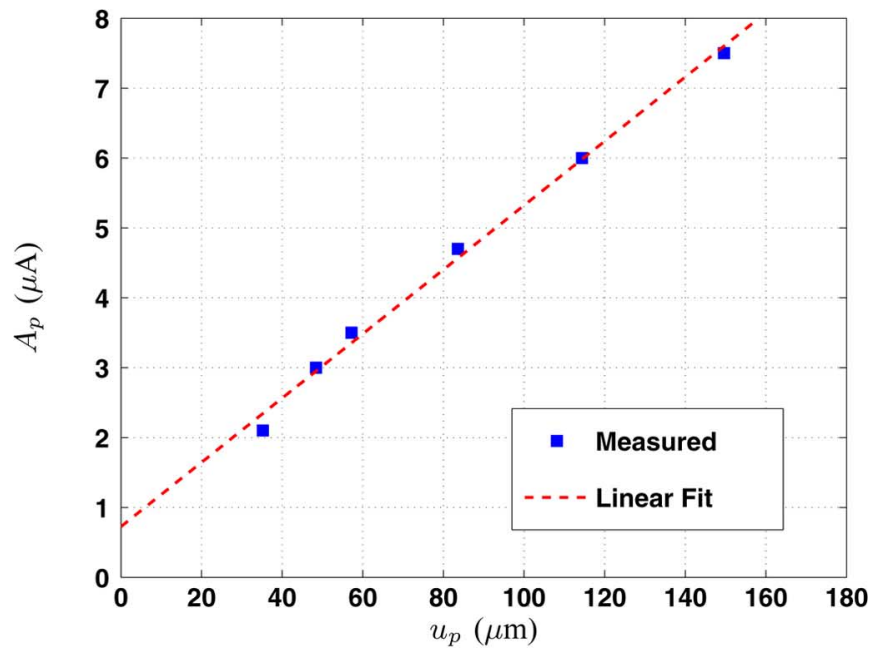

(a)

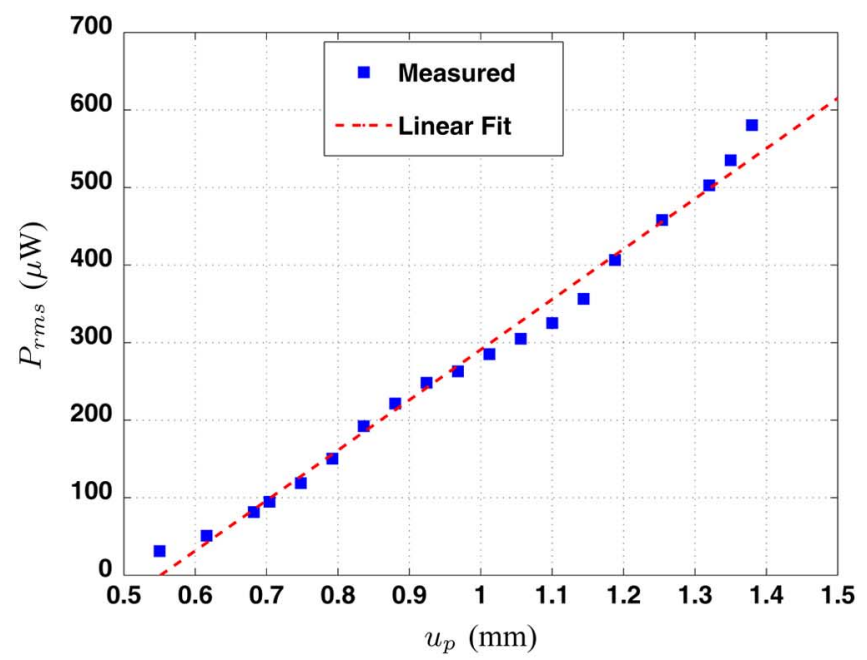

(b)

Fig. 8. (a) Short-circuit current amplitude (peak-to-peak) versus the base displacement amplitude (peak-to-peak). (b) Harvested power (RMS) under shortcircuit conditions versus the base vibration displacement amplitude (peak-topeak).

$\omega_{1}$. At $u_{p}=1.3 \mathrm{~mm}$, the output short-circuit power is around $P_{p}=0.5 \mathrm{~mW}$. When $u_{p}=1.3 \mathrm{~mm}$, the peak amplitude of the cantilever tip is estimated as

$$
w_{\mathrm{pp}}=\frac{A_{p} \omega_{1}^{2} u_{p}}{2 \zeta \sqrt{1-\zeta^{2}}}=8.1 \mathrm{~mm}
$$

The active device volume ${ }^{1}$ is then estimated as $V o_{1}=l b w_{\mathrm{pp}}=$ $1.205 \times 10^{-1} \mathrm{~cm}^{3}$. Therefore, the power density under the shortcircuit condition is then

$$
\mathrm{PD}_{1}=\frac{P_{p}}{V o_{1}}=4.15 \mathrm{~mW} / \mathrm{cm}^{3} \text {. }
$$

We only consider the short-circuit conditions in the power density estimation. With a large resistance load, the harvested power will increase [6], [9]. Therefore, the above estimation of the power density is conservative.

\footnotetext{
${ }^{1}$ The active device volume is defined as the occupied volume by the harvester under excitations [9].
}

\section{Natural Frequency of a $10 \mathrm{~mm}$ Cantilever}

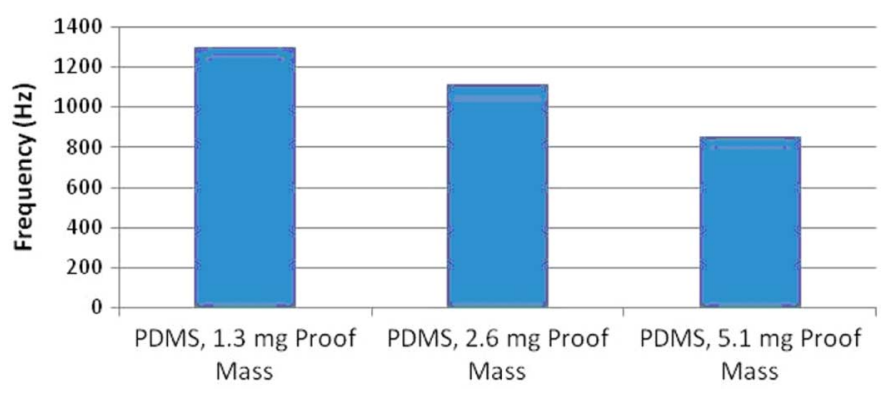

Fig. 9. Comparison of the first-mode natural frequencies with various sizes of the proof mass.

\section{PERformance COMPARISONS}

In this section, we analyze and compare the PMN-PT/PDMS harvester output power density performance. We first discuss a new harvester design of the same geometry as the prototype but with a larger proof mass $M_{\mathrm{pd}}$ than that of the prototype. The use of the large proof mass is to reduce the natural frequency of the harvester system. We then predict the performance of the new design based on the dynamic system model, and compare with an MEMS-based PZT harvester design reported in [9].

\section{A. Frequency Tuning}

For the PMN-PT/PDMS design, we use the proof mass to tune the resonant frequency of the cantilever structure. We have analyzed relationship (4) between the mass of the proof mass and the natural frequency. It has been shown that the increasing mass of proof mass decreases natural frequency of the harvester system. Fig. 9 shows experimental results of the first natural frequency variations under three different sizes of the proof mass. According to the analysis, the natural frequency will roughly decrease by $115 \mathrm{~Hz}$ for every milligram of mass placed on the tip of a $10 \mathrm{~mm}$ PMN-PT cantilever.

\section{B. Harvester Performance Comparison}

The output power of the harvester depends on the input vibration frequency and amplitude. The calculation of the power density for the harvester prototype in the previous section is under the vibration amplitude of $1.3 \mathrm{~mm}$ at the frequency around $1.3 \mathrm{kHz}$. To compare the PMN-PT/PDMS harvester performance with other reported harvesters, we need to evaluate the output of the harvester under a typical environmental vibration source. There are limited experimental data on harvester's energy density in the literature. Here, we take an example in [9] to evaluate the PMN-PT/PDMS harvester performance.

In [9], an MEMS-based PZT vibration energy harvester has been designed, and the output short-circuit power density is predicted around $28.2 \mu \mathrm{W} / \mathrm{cm}^{3}$. The power density prediction is based on the vibration source of a microwave oven side panel with an acceleration $4.2 \mathrm{~m} / \mathrm{s}^{2}$ at a frequency of $150 \mathrm{~Hz}$. The size of the MEMS-based PZT harvester in [9] is around $1.2 \mathrm{~mm} \times 0.5 \mathrm{~mm} \times 100 \mu \mathrm{m}$. Assuming the input vibration is harmonic, the excitation amplitude $u_{\text {ip }}$ is estimated as

$$
u_{\mathrm{ip}}=\frac{4.2}{(2 \pi \cdot 150)^{2}}=4.73 \mu \mathrm{m} .
$$


To reduce the natural frequency of the current design (around $\omega_{1}=1.3 \mathrm{kHz}$ ) to $\omega_{d}=150 \mathrm{~Hz}$, we only change the mass $M_{\mathrm{pd}}$ of the tip proof mass. In the prototype design, the mass of the proof mass is $M_{p 1}=\alpha_{1} m l=0.166 m l$ and $\lambda_{1}:=\lambda\left(\alpha_{1}\right)=$ 1.655 (Table II). Using (3), we have

$$
\lambda_{d}:=\lambda\left(\alpha_{d}\right)=\lambda_{1} \sqrt{\frac{\omega_{1}}{\omega_{d}}}=0.558
$$

where $\alpha_{d}$ is the mass ratio of the new harvester design. Using (4), we obtain $\alpha_{d}=30.75$, and the desired mass $M_{\mathrm{pd}}$ of the proof mass in the new harvester design is thus

$$
M_{\mathrm{pd}}=\alpha_{d} m l=250.3 \mathrm{mg} \text {. }
$$

For the new harvester design, the coefficients $A$ and $C$ in (8) are different with the current prototype due to the changing natural frequency $\lambda_{d}$ in (21). Therefore, generalized coefficients $m_{e}, c_{e}, k_{e}$, in (10), damping coefficient $\zeta$ and transfer function gains in (16) and (18) are all different with those of the prototype design. Since we have the closed-form formulation for these coefficients and parameters, we estimate the values of the new design from the prototype data.

Let $\zeta_{d}, A_{v d}$, and $A_{\mathrm{pd}}$ denote the damping ratio coefficient, voltage and tip displacement transfer function gains, respectively, under the new harvester design. We also denote $\zeta_{1}, A_{v 1}$, and $A_{p 1}$, respectively, for their values of the prototype harvester. From (11) and (12), we obtain

$$
\boldsymbol{\gamma}_{\zeta}:=\frac{\zeta_{d}}{\zeta_{1}}=\frac{g_{\zeta}\left(\alpha_{d}\right)}{g_{\zeta}\left(\alpha_{1}\right)}
$$

where function $g_{\zeta}$ is obtained as

$$
g_{\zeta}(\alpha):=a_{2}(\alpha) \sqrt{\frac{a_{1}(\alpha) \alpha+a_{2}(\alpha)}{a_{3}(\alpha)}} .
$$

Here, we explicitly write the dependency relationship between parameters $a_{i}, i=1, \ldots, 6$, and $\alpha$ through (9). Similarly, we obtain the relationships

$$
\gamma_{A_{v}}:=\frac{A_{v d}}{A_{v 1}}=\frac{g_{A_{v}}\left(\alpha_{d}\right)}{g_{A_{v}}\left(\alpha_{1}\right)} \quad \gamma_{A_{p}}:=\frac{A_{v p}}{A_{p 1}}=\frac{g_{A_{p}}\left(\alpha_{d}\right)}{g_{A_{p}}\left(\alpha_{1}\right)}
$$

where

$$
\begin{aligned}
g_{A_{v}}(\alpha) & :=\frac{a_{6}(\alpha)\left[a_{4}(\alpha) \alpha+a_{5}\right]}{a_{3}(\alpha)} \\
g_{A_{p}}(\alpha) & :=\frac{a_{4}(\alpha)}{a_{6}(\alpha)} g_{A_{v}}(\alpha) .
\end{aligned}
$$

Plugging $\alpha_{d}=30.75$ and $\alpha_{1}=0.16$ into (22) and (23), we obtain

$$
\boldsymbol{\gamma}_{\zeta}=0.0239, \boldsymbol{\gamma}_{A_{v}}=68.59, \boldsymbol{\gamma}_{A_{p}}=59.48
$$

From (19), the output peak voltage $V_{\mathrm{pd}}$ and the tip displacement $w_{\text {ppd }}$ of the new harvester design are, respectively

$$
V_{\mathrm{pd}}=\frac{\boldsymbol{\gamma}_{A_{v}}}{\boldsymbol{\gamma}_{\zeta}}\left(\frac{\omega_{d}}{\omega_{1}}\right)^{2} \frac{u_{\mathrm{ip}}}{u_{p}} V_{p 1}=1.75 \mathrm{~V}
$$

and

$$
w_{\mathrm{pd}}=\frac{\boldsymbol{\gamma}_{A_{p}}}{\gamma_{\zeta}}\left(\frac{\omega_{d}}{\omega_{1}}\right)^{2} \frac{u_{\mathrm{ip}}}{u_{p}} w_{p 1}=947.2 \mu \mathrm{m}
$$

For the short-circuit power density, we assume the output power $P=V_{p}^{2} / R_{h}$ under the short circuit condition, where $R_{h}$ is the magnitude of the impedance of the harvester. The impedance is assumed unchanged in the new design since only the proof mass is changed. Then, the output power density of the new design $\mathrm{PD}_{d}$ and that of the harvester prototype $\mathrm{PD}_{1}$ satisfy the following relationship

$$
\mathrm{PD}_{d}=\frac{\gamma_{A_{v}}^{2}}{\gamma_{\zeta} \gamma_{A_{p}}}\left(\frac{\omega_{d}}{\omega_{1}}\right)^{2} \frac{u_{\mathrm{ip}}}{u_{p}} \mathrm{PD}_{1}=645.3 \mu \mathrm{W} / \mathrm{cm}^{3} .
$$

The estimate of the power density of the new PMN-PT/PDMS harvester design is about 20 times larger than the prediction of the MEMS-based PZT micro-harvester in [9]. The high-power density of the design is mainly due to the fact that the use of the large proof mass reduces the stiffness of the beam/mass systems, and therefore increases the vibration magnitude under a smallamplitude input excitation.

\section{CONCLUSION}

In this paper, we designed, analyzed, and experimentally tested a vibration-based energy harvester that is made of a PMN-PT/PDMS composite beam. The motivation of this study is to achieve a high-energy conversion efficiency. The use of PMN-PT piezoelectric material and an IDEs design improved the energy conversion efficiency. The proof mass design at the cantilever tip is used as a means to tune the natural frequency of the harvester system. We also presented a dynamic systems modeling scheme to analyze the responses and performance of the harvester design. Based on the experiments on a prototype, we analyzed and predicted the output power density performance of the harvester design. The comparison study demonstrated a superior output power density performance than that of an existing reported harvesters.

We are currently refining our design to lower the natural frequency of the harvester and experimentally validate the analysis. Optimization and miniature design of the PMN-PT harvester and adaptively passive tuning the resonant frequency of the harvester structure are the other ongoing research directions.

\section{APPENDIX \\ Calculation of Peak Values of Responses of HARVESTER SYSTEM UNDER A STEP INPUT}

Under the step input $u(t)=u_{p} / s$, the response of the proof mass displacement at the tip of the beam is

$$
w_{p}(t)=\frac{A_{p} \omega_{n}^{2} u_{p}}{\sqrt{1-\zeta^{2}}} e^{-\zeta \omega_{n} t} \cos \left(\omega_{d} t+\phi\right) .
$$

Since the oscillation period is $2 \pi / w_{d}$, it is straightforward to calculate that the ratio $R_{i}$ as

$$
R_{i}=\frac{w_{p i}}{w_{p(i+1)}}=\exp \left(\frac{2 \pi \zeta}{\sqrt{1-\zeta^{2}}}\right)
$$

and the first equation in (20) follows directly. 
We take the derivatives of $w_{p}(t)$

$$
\begin{aligned}
& \dot{w}_{p}(t)=-\frac{A_{p} \omega_{n}^{3} u_{p}}{\sqrt{1-\zeta^{2}}} e^{-\zeta \omega_{n} t} \sin \left(\omega_{d} t+2 \phi\right) \\
& \ddot{w}_{p}(t)=-\frac{A_{p} \omega_{n}^{4} u_{p}}{\sqrt{1-\zeta^{2}}} e^{-\zeta \omega_{n} t} \cos \left(\omega_{d} t+3 \phi\right) .
\end{aligned}
$$

To obtain the $i$ th peak value at time $t_{i}, i=1,2, \ldots$, we need

$$
\dot{w}_{p}\left(t_{i}\right)=0 \text {, and } \ddot{w}_{p}\left(t_{i}\right)<0 \text {. }
$$

Then, we obtain $\omega_{d} t_{i}=2 i \pi-2 \phi$ and the $i$ th peak value of $w_{p}(t)$ is given as

$$
w_{p i}:=\left|w_{p}\left(t_{i}\right)\right|=\frac{A_{p} \omega_{n}^{2} u_{p} \cos \phi}{\sqrt{1-\zeta^{2}}} \exp \left(-\frac{\zeta(2 i \pi-2 \phi)}{\sqrt{1-\zeta^{2}}}\right) .
$$

Therefore, the second formula in (20) is obtained.

\section{ACKNOWLEDGMENT}

J. Yi thanks Dr. S. M. Shahruz at the Berkeley Engineering Research Institute for helpful discussions on vibration modeling of cantilever beams. The authors also thank two anonymous reviewers for their constructive suggestions.

\section{REFERENCES}

[1] S. Anton and H. Sodano, "A review of a power harvesting using piezoelectric materials (2003-2006)," Smart Mater. Struct., vol. 16, no. 3, pp. R1-R21, 2007.

[2] R. Amirtharajah and A. Chandrakasan, "Self-powered signal processing using vibration-based power generation," IEEE J. Solid-State Circuits, vol. 33, no. 5, pp. 687-695, 1998.

[3] P. Glynne-Jones, S. Beeby, and N. White, "Towards a piezoelectric vibration-powered microgeneration," IEE Proc.-Sci. Meas. Technol., vol. 148, no. 2, pp. 68-72, 2001.

[4] P. Mitcheson, T. Green, E. Yeatman, and A. Holmes, "Architectures of vibration-driven micropower generators," J. Microelectromech. Syst., vol. 13, no. 3, pp. 429-440, 2004.

[5] S. R. Platt, S. Farritor, and H. Haider, "On low-frequency electric power generation with PZT ceramics," IEEE/ASME Trans. Mechatron., vol. 10, no. 2, pp. 240-252, 2005.

[6] Y. Jeon, R. Sood, J. Jeong, and S. Kim, "MEMS power generator with transverse mode thin film PZT," Sens. Actuators, A, vol. 122, pp. 16-22, 2005.

[7] S. Roundy, "On the effectiveness of vibration-based energy harvesting," J. Intell. Mater. Syst. Struct., vol. 16, no. 10, pp. 809-823, 2005.

[8] S. Roundy, P. Wright, and J. Rabaey, Energy Scavenging for Wireless Sensor Networks: With Special Focus on Vibrations. Boston, MA: Kluwer Academic, 2004.

[9] N. duToit, B. Wardle, and S. Kim, "Design considerations for MEMSscale piezoelectric mechanical vibration energy harvesters," Integ. Ferroelec., vol. 71, no. 1, pp. 121-160, 2005.

[10] W. Choi, Y. Jeon, J. Jeong, R. Sood, and S. Kim, "Energy harvesting MEMS device based on thin film piezoelectric cantilevers," J. Electroceramtics, vol. 17, no. 2-4, pp. 543-548, 2006.

[11] S. Shahruz, "Design of mechanical band-pass filters for energy scavenging," J. Sound Vib., vol. 292, no. 3-5, pp. 987-998, 2006.

[12] Y. Shu and I. Lien, "Analysis of power output for piezoelectric energy harvesting systems," Smart Mater. Struct., vol. 15, no. 6, pp. 1499-1512, 2006.

[13] Y. Hong and K. Moon, "Single crystal piezoelectric transducers to harvest vibration energy," in Proc. SPIE, Sapporo, Japan, 2005, paper number 6048E1.

[14] Y. Hong, K. Moon, M. Levy, and R. Vanga, "Single-crystal film piezoelectric sensors, actuators and energy harvesters with interdigitated electrodes," Ferroelectrics, vol. 342, pp. 1-13, 2006.
[15] K. Moon, H. Liang, J. Yi, and B. Mika, "Tire tread deformation sensor and energy harvester development for "smart tire" applications," in Proc. SPIE, San Diego, CA, 2007, paper number 65290K.

[16] S. Park and T. Shrout, "Ultrahigh strain and piezoelectric behavior in relaxor based ferroelectric single crystals," J. Appl. Phys., vol. 82, pp. 1804-1811, 1997.

[17] V. Giurgiutiu and S. Lyshevski, Micromechatronics: Modeling, Analysis, and Design with MATLAB. Boca Raton, FL: CRC Press, 2004.

[18] R. Clough and J. Penzin, Dynamics of Structures. New York: McGraw-Hill, 1993.

[19] S. Mahmoodi, N. Jalili, and M. Daqaq, "Modeling, nonlinear dynamics, and identification of a piezoelectrically actuated microcantilever sensor," IEEE/ASME Trans. Mechatron., vol. 13, no. 1, pp. $58-65,2008$.

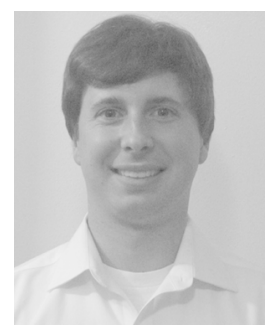

Alex Mathers received the B.S. degree in integrated science and technology from James Madison University, Harrisonburg, VA, in 2003 and the M.S. degree in mechanical engineering from San Diego State University, San Diego, CA, in 2008.

$\mathrm{He}$ is currently a Mechanical Engineer for Qualcomm Inc. His research includes the design and fabrication of mechatronic and MEMS devices.

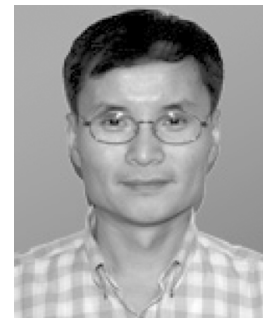

Kee S. Moon received the Ph.D. from the University of Illinois, Chicago.

In 2005, he joined the faculty of San Diego State University, San Diego, CA, where he is currently an Associate Professor of Mechanical Engineering. Before joining San Diego State University, he was an Associate Professor in the Department of Mechanical Engineering-Engineering Mechanics, Michigan Technological University. He has authored more than 100 published technical papers. His primary research interests are in smart sensor/actuator technology and integrated systems.

Prof. Moon received the Outstanding Faculty Award in 2007 from San Diego State University. He is a member of The International Society for Optical Engineers (SPIE).

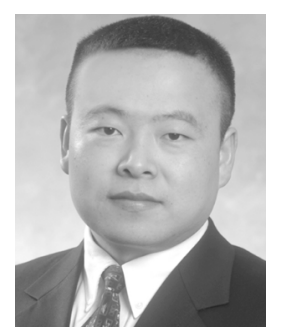

Jingang Yi (S'99-M'02-SM'07) received the B.S. degree in electrical engineering from the Zhejiang University, Hangzhou, China, in 1993, the M.Eng. degree in precision instruments from Tsinghua University, Beijing, China, in 1996, the M.A. degree in mathematics, and the Ph.D. degree in mechanical engineering from the University of California, Berkeley, in 2001 and 2002, respectively.

$\mathrm{He}$ is currently an Assistant Professor of Mechanical Engineering at Rutgers University. Prior to joining Rutgers University in August 2008, he was an Assistant Professor of Mechanical Engineering at San Diego State University since January 2007. From May 2002 to January 2005, he was with Lam Research Corporation, Fremont, CA, as a member of Technical Staff. From January 2005 to December 2006, he was with the Department of Mechanical Engineering, Texas A\&M University, as a Visiting Assistant Professor. His research interests include autonomous robotic systems, dynamic systems and control, intelligent sensing and actuation systems, mechatronics, automation science and engineering, with applications to semiconductor manufacturing, intelligent transportation, and biomedical systems.

Dr. Yi is a member of the American Society of Mechanical Engineers (ASME). He currently serves as an Associate Editor of the ASME Dynamic Systems and Control Division and the IEEE Robotics and Automation Society Conference Editorial Boards. He also serves as a Guest Editor of IEEE TRANSACTIONS ON AUTOMATION SCIENCE AND ENGINEERING. 